\title{
Outcome riproduttivi e perinatali in donne con iperplasia surrenalica congenita: studio di coorte
}

\author{
Maria Chiara Di Carlo ${ }^{1} \cdot$ Emanuela Arvat $^{1}$
}

Accettato: 7 settembre 2021 / Pubblicato online: 7 febbraio 2022

(c) The Author(s) 2022

\section{Commento a:}

Reproductive and perinatal outcomes in women with congenital adrenal hyperplasia: a population-based cohort study.

A.L. Hirschberg, S. Gidlöf, H. Falhammar, L. Frisén, C. Almqvist, A. Nordenskjöld, A. Nordenström.

J Clin Endocr Metab (2021) 106(2):e957-e965

L'iperplasia surrenalica congenita $(\mathrm{CAH})$ è un gruppo di malattie genetiche caratterizzato da disordini ereditari di tipo recessivo causati da difetti enzimatici della steroidogenesi surrenalica e gonadica, con incidenza di 1:14.000-18.000 nati.

Nelle forme più frequenti, i precursori ormonali accumulati vengono deviati verso la produzione di androgeni, causandone un eccesso, associato alla compromissione completa o parziale della sintesi di cortisolo, aldosterone, o entrambi. In alcune forme più rare, è insufficiente anche la sintesi di androgeni. Nelle forme in cui la produzione di cortisolo è assente o diminuita, la liberazione di ormone adrenocorticotropo (corticotropina, ACTH), normalmente soppressa dal cortisolo, è aumentata. Le forme più comuni di iperplasia surrenalica congenita sono il deficit di 21-idrossilasi e il deficit di 11beta-idrossilasi. In queste forme, i precursori prossimali al blocco enzimatico si accumulano e sono convogliati verso la sintesi di androgeni surrenalici. La conseguente eccessiva secrezione di androgeni causa virilizzazione di vario grado nei genitali esterni delle femmine; nessun difetto è percepibile nei genitali esterni dei maschi. Nel deficit di 21-idrossilasi si distinguono due tipi principali: una forma classica, che può essere virilizzante semplice o con perdita di sali e una forma non classica in cui la produzione di cortisolo, normale o deficitaria, viene resa in evidenza solo dopo test da stimolo con ACTH $250 \mathrm{mcg}$.

\section{E. Arvat}

emanuela.arvat@unito.it

1 Endocrinologia, Diabetologia e Metabolismo, Dipartimento di Scienze Mediche, Università degli Studi di Torino, Torino, Italia
Numerosi studi sulla fertilità sono stati condotti sulle femmine affette da CAH ed è, quindi, ormai noto in letteratura come le donne con $\mathrm{CAH}$, soprattutto nelle forme classiche con perdita di sali, mostrino ridotta fertilità rispetto alla popolazione generale, una riduzione del numero di gravidanze e di parti e un aumento del tasso di aborto, quest'ultimo ancor più se la paziente non assume terapia glucocorticoide. La ridotta fertilità nella donna, soprattutto nella forma classica, risente di diversi meccanismi alla base; i due principali riportati in letteratura sono l'aumento degli androgeni e l'aumento dei progestinici surrenalici.

Tuttavia, pochi studi hanno effettivamente indagato gli outcome riproduttivi e perinatali in queste donne.

A tal proposito, Hirschberg e colleghi [1] hanno condotto un'analisi mirata a valutare gli outcome riproduttivi e perinatali nelle donne affette da $\mathrm{CAH}$, cercando di riunire i dati finora raccolti in materia ed eseguendo uno studio di coorte sulla popolazione svedese. Nello studio sono stati reclutati, tramite i registri nazionali svedesi, un totale di 272 donne con CAH da deficit della 21-idrossilasi e 27.200 controlli, appaiati per sesso, età (mediana 31 anni) e luogo di nascita. In particolare, lo studio valutava la proporzione di donne con CAH che conducevano a termine la gravidanza fino alla nascita e gli outcome riproduttivi e perinatali.

Dallo studio si evince come, delle 272 donne con CAH, solo 69 hanno dato vita almeno a un figlio $(25,4 \%)$, una frequenza più bassa rispetto ai controlli $(45,8 \%, p<0,001)$. Inoltre, donne con CAH avevano meno figli rispetto ai controlli e risultavano avere un'età lievemente maggiore alla nascita del loro primo figlio. Più donne con $\mathrm{CAH}$ hanno ricevuto diagnosi di diabete gestazionale rispetto ai controlli $(4,9$ vs $1,4 \%, p<0,05)$ e sono andate maggiormente incontro a parto cesareo $(51,4$ vs $12,3 \%, p<0,05)$, probabilmente per multipli interventi di chirurgia di ricostruzione dei genitali. Diversamente, non è stata riscontrata nessuna differenza statisticamente significativa nell' Apgar score tra casi e controlli, così come per la frequenza di nati piccoli per età gestazionale, morti alla nascita, circonferenza del 
capo e lunghezza, tra figli nati da madri con CAH e controlli. Non è stata riscontrata differenza significativa nel tasso di incidenza di pre-eclampsia tra casi e controlli.

In conclusione, si può affermare da questo studio che il tasso di nascite sia inferiore nelle donne con $\mathrm{CAH}$ rispetto alla popolazione generale e la diagnosi di diabete gestazionale e il parto tramite taglio cesareo risultano più frequenti; tuttavia, gli outcome perinatali sono comparabili ai controlli.

Studi futuri dovrebbero includere, di conseguenza, più dettagliate informazioni sulle tecniche di riproduzione assistita in queste pazienti e porre l'attenzione su eventuali fattori di rischio per lo sviluppo di diabete gestazionale. Ancora, con lo sviluppo futuro e la produzione di nuove formulazioni di corticosteroidi usati nella terapia della sindrome adrenogenitale, si potrà ambire a una farmacocinetica tale da mimare la corretta fisiologica dell'asse ipotalamo-ipofisi-surrene portando, quindi, a un miglioramento del compenso di malattia, a una riduzione delle comorbidità associate alla terapia steroidea e, contestualmente, a una riduzione della subfertilità che caratterizza queste donne, con miglioramento degli outcome riproduttivi.
Open Access This article is licensed under a Creative Commons Attribution 4.0 International License, which permits use, sharing, adaptation, distribution and reproduction in any medium or format, as long as you give appropriate credit to the original author(s) and the source, provide a link to the Creative Commons licence, and indicate if changes were made. The images or other third party material in this article are included in the article's Creative Commons licence, unless indicated otherwise in a credit line to the material. If material is not included in the article's Creative Commons licence and your intended use is not permitted by statutory regulation or exceeds the permitted use, you will need to obtain permission directly from the copyright holder. To view a copy of this licence, visit http://creativecommons.org/licenses/by/4.0/.

\section{Bibliografia}

1. Hirschberg AL, Gidlöf S, Falhammar H et al (2021) Reproductive and perinatal outcomes in women with congenital adrenal hyperplasia: a population-based cohort study. J Clin Endocr Metab 106(2):e957-e965

Nota della casa editrice Springer Nature rimane neutrale in riguardo alle rivendicazioni giurisdizionali nelle mappe pubblicate e nelle affiliazioni istituzionali. 